\title{
CORRECTION
}

\section{Correction to: Gestational age, sex, and time affect urine biomarker concentrations in extremely low gestational age neonates}

David J. Askenazi, Brian A. Halloran, Patrick J. Heagerty, Robert H. Schmicker, Patrick Brophy, Sandra E. Juul, Sangeeta Hingorani, Stuart L. Goldsteinon behalf of the PENUT Trial Consortium

(c) The Author(s), under exclusive licence to the International Pediatric Research Foundation, Inc 2021

Pediatric Research (2022) 92:334; https://doi.org/10.1038/s41390-021-01898-5

Correction to: Pediatric Research https://doi.org/10.1038/s41390021-01814-x, published online 30 November 2021
Due to a technical problem, the author name Brian Halloran was not displayed in the author list of the PDF version of this article. The original article has been corrected. 